\title{
Abstract Author Index
}

A

Abraham, Ivo ..................P07.07, P07.08 Abrams, Donald...............P05.20, P07.56

Acquah, Joseph .OA05.04

Acree, Michael ................P05.01, P09.31

Acworth, Edward........................... P03.24

Adams, Alyssa ............................ P05.16

Adelstein, Pamela ........................ P05.39

Adler, Elizabeth........................... P05.01

Adler, Shelley ............................. P07.56

Agarwal, Gaurava ........................ P07.32

Agarwal, Shashi............ P05.02, P05.03, P05.04, P05.05

Agcaoili, Gary OA13.04

Ahmadian, Mohammad ............... P07.44

Ahrens, Isabella ............................ P07.01

Ahrns, Caleb ................................ P09.16

Akagi, Hiroko ............................... P07.29

al Haddabi, Atheer ....................... P07.29

AlBedah, Abdullah ...................... P07.48

Alcantara, Joel ...............P04.14, P04.18, P04.19

Alcantara, Junjoe ...........P04.14, P04.18, P04.19

Alexander, Joshua........................ P04.07

Alexander, William ..................... P04.13

Ali, Ather................OA04.04, OA07.01,

OA08.02, P01.01, P05.06, P09.01,

P09.03, P09.29

Altman, Doug G.

..OA06.02

Altschuler, Andrea....................... P08.06

Amick, Halle .................................. P07.02

Amri, Hakima ................................ P09.24

Anderson, Belinda ..........P06.09, P08.04

Anderson, David............................ P05.69

André, Karyn BS ......................... P05.48

Andresen, Andrew .....................OA03.02

Anheyer, Dennis .......................... P05.17

Apollo, Damian . OA04.04 P09.01

Arce, Lauren . $\mathrm{P} 05.23$

Arnedt, John Todd OA05.01

Arzola, Sonya

P09.02

Asher, Gary................................... P07.02

Atchley, Rachel ... P09.04

Atreya, Chloe................................ P08.06

Attias, Samuel................P02.25, P03.11, P04.01

Aucoin, Monique ............P03.01, P05.08

Auerbach, Stephen....................... P06.15

Aveni, Eleonore ........................... P07.54

Avins, Andrew ................P05.69, P08.06

Avnery, Ofri ................................ P04.01

Avshalomov, David...................... P02.25

Azhar, Salman

P08.04
B

Bacchetti, Peter..............P05.01, P09.31

Baek, Seon-Eun ............................ P09.36

Baek, Yun Hui............................ P07.05

Bahrami Taghanaki,

Hamid Reza

$\mathrm{P} 07.44$

Bai, Wenjing.

P09.25

Bair, Matthew J .............P04.09, P04.10

Bakosh, Laura

OA15.03

Ballabeni, Pierluigi ......................... P07.54

Balneaves, Lynda G .......P03.01, P03.15

Bann, Carla

$\mathrm{P} 07.02$

Bao, Ting

P02.01

Bao, Tuya ......... P01.13, P01.24, P01.27

Bao, Wuye ..................................... P01.09

Barber, Melissa............................ P08.01

Barbour, Linda............................ P09.30

Bardacke, Nancy......................... P05.63

Barnes, Patricia............................. P05.73

Barrows, Kevin ......................... OA05.04

Barth, Jürgen............................. OA06.02

Bassett, Sarah ............................. P05.65

Batchelder, Abigail...................... P05.65

Batisti, Ana P ..............................P01.16

Bauer, Brent................................ P07.54

Baumann, Nora ............................ P04.03

Bayley, Peter................................ P05.07

Bechard, Elizabeth...................... OA07.01

Bedregal, Paula........................... P07.03

Beljanski, Sylvie.......................... P03.02

Bell, Iris R

OA15.01

Belmonte, Luana M.......................P09.06

Belmonte, Luiz AO ..................... .P09.06

Ben Arye, Eran ........................... P03.11, P04.01

Ben-Ami, Sarah ........................... P02.04

Ben-David, Merav ........................ P02.04

Benes, Lane ................................... P04.11

Benjamin, Ellen ............................. P04.10

Bensoussan, Alan ....................... P01.34

Bergman, Aaron ........................OA01.02

Berman, Brian M....OA06.02, OA10.01, P01.26

Berman, Sue

OA10.01

Beroes, Jessica ..................P05.60, P07.30

Bertisch, Suzanne ....................... P05.22

Bhadkamkar, Nishin ................... P07.25

Bhasin, Manoj................................. P01.02

Bingcong, Zhao ......................... P08.17

Birdee, Gurjeet ........... OA15.02, P07.01

Bishop, Felicity.......... OA08.03, P07.26, P08.08, P09.10

Bitton, Kohava.

$\mathrm{P} 02.25$

Bjerke, Wendy

P05.24

Black, David
Blackburn, Elizabeth ......P05.49, P05.64

Blair, Janis .................................... P07.18

Blashill, Aaron............................. P05.42

Blödt, Susanne ........................... OA05.02

Bo, Xiangwei ................................ P01.29

Bokhour, Barbara ...................... OA10.04

Boland, Erin................................... P07.02

Bolton, Rendelle ........................ OA10.04

Bonnell, Sian ..................P07.47, P08.14

Bonvin, Eric................................... P07.54

Boon, Heather............................. P07.17

Boone, Jill ...................................... P09.05

Borno, Hala................................... P08.06

Boschmann, Michael ................... P03.12

Boyd, Courtney........................... P07.06

Boysen, James .............................. P04.13

Bradley, Constance....................... P07.18

Bradley, Ryan .................P03.03, P08.01

Branda, Megan ............................. P07.40

Braun, Sarah .... P05.09, P05.52, P06.15

Breau, Rodney ............................. P03.01

Brendler, Charles ........................... P05.59

Brewer, Judson ............................. P05.10

Brewer, Lauren ............................ P01.08

Brittan, Kathleen .......................... P07.36

Brodsky, Marc ............................. P07.04

Brooks, Audrey.......................... OA08.01

Brooks Holliday, Stephanie ........ P05.16

Brown, Richard........... OA02.04, P05.58

Bu, Xiangwei ................................ P01.33

Bull, Sheana................................. P09.30

Buring, Julie .............. OA03.03, P07.33

Burnand, Bernard ...........P07.09, P07.35

Burnett-Zeigler, Inger................... P05.66

Burns, Jacquelyn........................... P09.26

Burns, Mark ................................ P07.18

Busch, Martine ..........................OA03.01

Butzer, Bethany ......................... P05.32

Byerly, Laura ............................... P06.01

Bénini, Patrice ............................ P04.17

Büssing, Arndt ................................ P05.53

C

Cahn, B. Rael ................................ P05.71

Caines, Laurie .............................. P06.17

Caldwell, Patrina Ha Yuen ......... P09.11

Call, Stephanie............................... P06.15

Cambron, Jerrilyn ........................ P06.09

Cao, Huijuan ................................... P03.04

Cao, Jin ...................................... P01.31

Carim Todd, Laura ....................... P05.11

Carmel, Narin ............................... P02.04

Carola, Mary ................................. OA07.01

Carraro, Emerson........................... P01.36 
Carrico, Adam ............................. P05.65

Carrico, Caroline ............P05.09, P05.52

Carson, James .................P05.12, P05.13

Carson, Kimberly .......................... P05.12

Cassidy, J David........................OA06.03

Catane, Raphael ............................ P02.04

Ceci, Lisandro................................ P01.36

Chad-Friedman, Emma ..P05.18, P06.01

Chae, Heechin.............................. P05.73

Chai, Qian-Yun............................. P02.28

Chalder, Trudie............................. P07.27

Chamberland, Paul ....................... P09.18

Chan, Noel Yanki......................... P01.02

Chang, Alexandra ......................OA05.04

Chang, Bei .................................... P05.21

Chang, Dennis ............................. P01.34

Chang, Mayling ........................... P07.03

Chao, Maria T ............ OA05.04, P02.22

Chaoul, Alejandro.......................... P05.77

Chaukos, Deanna .......................... P06.01

Chen, Chen .................................. P09.27

Chen, Jianxin ................................ P01.28

Chen, Kevin .................................. P02.02

Chen, Ni-ni ................................... P09.11

Chen, Ping ........ P01.03, P01.04, P01.05

Chen, Qi ..........................P01.03, P01.04, P01.05, P03.20

Chen, Wei ................................... P08.02

Chen, Yi....................................... P01.33

Cheng, Hsiang-Hung .................... P03.05

Cheng, Ke ................................... P01.26

Cheng, Ying Wu........................OA09.03

Cheng, Yu ..................................... P02.33

Chengcheng, Zhang ...................... P01.35

Cheong, Pui Kuan ......................... P08.12

Cheshire, Anna ........................... P08.04

Chesney, Margaret..OA10.01, OA13.04, P05.69

Chiaramonte, Delia....................OA10.01

Ching, Jessica ............................... P08.12

Chinh, Kelly ................................. P05.50

Cho, Hyun-Woo .......................... P02.23

Cho, Rachael................................. P05.07

Cho, Yu-Min.................................. P03.05

Choi, Byunghee ............................ P07.05

Choi, Koh-eun .............................. P07.19

Choi, Songie ...................P03.19, P08.03

Choi, Tae-Young ........................ P02.37

Chopra, Deepak ..............P05.49, P05.64

Chou, Chih-Ping ........................... P07.22

Christelis, Nick ............................. P09.10

Christianson, Jon .......................... P07.37

Christopher, Michael .................... P05.14

Chung, Vincent CH.......P02.30, P03.15, P03.17, P08.11, P08.12

Cidral-Filho, Francisco J.........OA06.04, P01.06, P01.16, P05.46, P05.48, P09.06

Citkovitz, Claudia.......................... P08.04

Cizmic, Vladislav ......................... P01.07

Cohen, Ezra ….............................. P05.15

Cohen, Lorenzo …......................... P05.77

Cohn, Michael ................P05.63, P05.65

Coker-Schwimmer, Emmanuel ... P07.02

Colgan, Dharmakaya .

P05.14
Connelly, Mark. P04.04, P05.33, P09.32

Cook, Joseph..................P02.22, P05.63

Cook, Karon .................................. P07.32

Cooley, Carl.................................. P07.36

Cooley, Kieran................P03.01, P05.08

Cordeiro, Eduarda........................ P09.19

Cordero, Evelyn.......................... OA15.03, P05.44

Corn, Joshua ...............................OA08.02

Cornelio-Flores, Oscar ................ OA07.02

Cotton, Sian .................................. P09.05

Coulter, Ian ...............................OA 10.03

Courtney, Rena ............................. P05.16

Cox, Diane ..................................... P07.29

Cramer, Holger .......OA07.03, OA09.01,

OA09.03, P05.17, P05.35

Crawford, Cindy

P07.06

Crocker, Heidi

$\mathrm{P} 06.14$

Crocker, Robert L............P07.07, P07.08

Crooks, Denise

Crowell, Sheila .....................

Cui, Yingxue ................................ P02.39

Curlin, Farr

P07.25

\section{D}

D'Adamo, Chris ......OA04.02, OA06.02 D'Urso, Antonietta ....................OA04.02

da Cruz, João N. M........................P09.06

da Silva, Morgana D .................. P01.06

Dallman, Mary............................. P09.31

Daubenmier, Jennifer .....P05.01, P09.31

Davies, Amber

$\mathrm{P} 04.10$

Davis, Jean

$\mathrm{P} 05.25$

Davis, Roger B .......OA03.03, OA15.01, P05.29

Dawson, Valerie

OA04.02

Dearing, Joshua ........................... P02.01

Decosterd, Isabelle .........P07.35, P07.54

Decoux, Brandi............................ P01.08

Deeb, George ...................P05.09, P05.52

Degoumois, Florence................... P07.35

Deiner, Stacie .............................. P05.75

Delagran, Louise........................... P06.02

Delebinski, Catharina .................... P01.19

Delibasic, Marija ......................... P04.05

Delitto, Anthony ........................OA03.04

Deng, Haiping................................ P01.26

Denninger, John W........P01.02, P05.18, P06.01

Desbiens, Jean-François ............... P06.08

Desrosiers, Johanne ..................... P07.31

DeVries, Renee. P06.02, P06.09, P06.11

Dhiman, Kartar ........................ OA09.04

Dhruva, Anand . P05.01, P07.56, P08.06

Dick, Andrew .............................OA06.01

Dieppe, Paul ..... P07.47, P08.14, P09.17

Diers, Tiffiny ............................... P09.05

Dmitrieva, Natalia ....................OA07.01

Dobos, Gustav ........OA07.03, OA09.01, OA09.03, P04.03, P05.17, P05.35

Dodds, Nathan ............................ P09.35

Dolor, Rowena............. OA11.02, P05.20

Dong, Fei .......... P01.15, P01.17, P02.17

Dong, Ruochen. P01.03, P01.04, P01.05

Dongmei, Duan.

$\mathrm{P} 08.17$
Doolin, Jim . P07.56

Doraiswamy, Murali.......P05.49, P05.64

Dossett, Michelle L .... OA15.01, P05.18

Dow, Alan...................................... P05.09

Dowler, Kristina ......................... P05.73

Dreusicke, Mark ......... OA01.03, P05.19

Driban, Jeffrey B ......................... .P05.45

Drisko, Jeanne ................P01.03, P03.20

Ducrest, Isabelle .......................... P07.09

Duncan, Larissa ............................. P05. P05.65

Dunlap, Corina ............................ P09.13

Dusek, Jeffery .........OA10.02, OA11.03,

OA11.04, P05.21, P05.32, P07.01,

P07.10, P07.37, P07.46, P07.49,

P07.52, P07.53, P08.15, P09.16

Dusek, Kathryn ............................. P05.21

Dutton, Anne .............................. P05.06

Dvorin, Kelly ...............................A10.04

E

Edelhäuser, Friedrich......P06.16, P06.18

Edman, Joel ................................. P05.20

Edmundson, Elizabeth................. P09.32

Eghbali, Babak ............................ P07.44

Eickhoff, Christine ....................... P05.16

Eiriksdottir, Ingibjorg ................. P05.30

Eisenberg, David ....OA03.03, OA11.01, P07.24, P07.33

Eisenhart-Rothe, Sanna .............OA05.02

Ekman, Eve.................................... P06.19

Elder, Charles ............................... P09.13

Elder, Nancy ................................ P09.05

Elder, William .............................. P04.15

Elsenbruch, Sigrid .....................OA09.01

Elwy, A. Rani ............................... P05.37

Enad, Racquel.............................. P05.38

Engle, Rosalinda.......................... P05.77

Engler, Harald............................ OA09.01

Ennis, Julie ..................................... P09.18

Eom, Tae-min ............................. P07.19

Epel, Elissa ...... P05.01, P05.49, P05.64, P09.31

Erickson, Lauren.

P05.21

Erlandsen, Andrew ..........P03.03, P09.15

Esmaaily, Habib .......................... P07.44

Estores, Irene ..................P02.02, P05.23

Eubanks, Robin........................... P06.06

Evans, Roni....................P06.02, P06.10

Eyler, Steven................................. P05.68

\section{F}

Falkenberg, Torkel ...................... P07.12

Fan, Yixin .................................... P01.37

Fang, John................................... P07.22

Fanshawe, Thomas ...................... P09.10

Farag, Christine ............................. P06.17

Farahmand, Kazem...................... P07.44

Farias, Miguel............................. P08.14

Farrell-Riley, Christine ................. P05.39

Fazekas, Anna............................... P09.18

Fazil, Mohd.....................................P07.13

Fehr, Johannes ............................OA09.03

Fei, Yu-Tong .... P02.03, P02.28, P07.42

Feistel, Björn .............................. P03.06

Femia, Alexandra ...........P05.22, P05.37 
Feng, L. P09.34

Feng, Shuo... P03.14, P09.11

Fengxian, Meng P01.35

Fergusson, Dean

P03.01

Fetterolf, Michael P09.35

Feuerstein, Joseph........................ P05.24, P06.03

Fields, Maxine ............................. P09.29

Figueiredo, Jane ............................ P07.23

Finch, Michael........OA11.03, OA11.04, P07.11, P07.46, P07.53, P08.15

Finnell, John

P09.33

Finti, Guillaume.

$\mathrm{P} 07.54$

Fletcher, Carol ............................ P07.10

Fleming, Jake................................ P04.07

Flores, Elizabeth .......................... P09.22

Florindez, Daniella ...................... P05.62

Flower, Andrew ….........P02.14, P07.26, P07.28, P08.08, P08.09

Fonsêca, Nina ............................. P04.17

Forneris, Catherine ...................... P07.02

Forrestal, John ............................ OA07.02

Fortney, Luke .............................. P04.11

Fouladbakhsh, Judith................... P05.25

Fox, Kealoha................................. P05.26

Freeland, Emilee............................ P04.09

Fritsma, Jolanda.........................OA03.01

Fritz, Heidi

P03.01

Fu, Liang-Wu

$\mathrm{P} 01.30$

$\mathrm{Fu}$, Rochelle.

OA13.01

Funabashi, Martha P04.12

Furusyo, Norihiro

$\mathrm{P} 02.35$

Fyfe-Johnson, Amber $\mathrm{P} 05.21$

\section{G}

Gaboury, Isabelle

$\mathrm{P} 07.31$

Gaines, Bradley ..... $\mathrm{P} 07.02$

Gainski Danielski, Lucinéia.....OA06.04

Gamba, Lygea F.

P05.48

Gamus, Dorit

$\mathrm{P} 02.04$

Gao, Xiao

P09.20

Garcia-Martinez, Irma .................. P01.01

Gardiner, Paula P05.39

Gascon, Gregg .P05.27, P05.38,

Gaylord, Susan

OA01.01

Gerbarg, Patricia......... OA02.04, P05.58

Gerent, Priscila M .......................P09.06

Gergen Barnett, Katherine .......... P05.39

Geronimo, Chris ........................ OA13.04

Gertlehner, Gerald ....................... P07.02

Ghooray, Kala............................... P09.22

Gibbs, Roz .................................. P08.09

Gilbert, Susan ….......................... P09.30

Ginex, Pamela................................. P09.26

Glaser, Dale ................................ P09.02

Glick, Ronald..................P02.33, P09.35

Go, Namgyeong........................... P02.34

Goertz, Christine......... OA03.02, P04.13, P07.36

Goff, Brandon... P09.02

Goldberger, Wendy ..................... P05.69

Goldenberg, Joshua ..................... P03.22

Goldhamer, Alan

P09.33
Goldingay, Sarah

P07.47, P08.14

Goldstein, Lee................................ P03.11

Goldstein, Lizabeth A ...............OA13.04

Gomes, Jossinelma ....................... P01.36

Goodrich, Elena.......... OA13.01, P05.11

Gorman, Peter .............................. P02.02

Gosselin, Donna ........................... P07.36

Gould-Fogerite, Susan ..............OA07.01, P06.06

Gow, Brian

OA03.03

Gowan-Moody, Donelda ............. P07.14

Graham, Cynthia.......................OA08.03

Graybill, Robert........................... P09.15

Greco, Carol ..... P02.33, P07.55, P09.35

Greeson, Jeffrey........................... P05.20

Grettie, Jessica ............................... P04.11

Greville-Harris, Maddy .............OA08.03

Griffin, Kristen .......... OA11.04, P07.11, P07.37, P07.46, P07.52, P07.53,

$\mathrm{P} 08.15, \mathrm{P} 09.16$

Grimerg, Ofra P04.01

Grimshaw, Jeremy ......................OA06.02

Grizzle, Amy J ...............P07.07, P07.08

Groessl, Erik …...........................OA13.03

Grosjean, Daniel ......................... P01.17

Gross, Cynthia R ..........................P05.28

Gu, Xiaohong ... P01.15, P01.17, P02.17

Guerrera, Mary ............................. P06.17

Guerra, Ximena ............................ P07.03

Guiterrez, Bruriah........................ P05.44

Gunnarsdottir, Salome ................. P07.15

Gunnarsdottir, Thora ...................P05.30,

P07.15

Guo, Tianwei

P02.05

Guo, Xiangyu

$\mathrm{P} 01.37$

Guo, Xuan.................................... P01.37

Guo, Yu ........... P01.09, P01.13, P01.27

Guo, Zhi-Ling ............................. P01.30

Guo, Zhong-Ning ....................... P02.28

Guo, Zhuo

$\mathrm{P} 01.24, \mathrm{P} 02.05$

Gupta, Shivenarain ..................... OA09.04

Gurvich, Olga ............................ P05.28

Gutierrez, Bruriah.....................OA15.03

Görne, Rainer C

P03.06

\section{H}

Haas, Mitch OA07.04, P06.10

Hafner, Timo .............................. OA02.03

Haller, Heidemarie ..... OA07.03, P04.03

Hamaguchi, Takuya...................... P01.10

Hampson, Johnson ...................... OA02.01

Han, Li ...........................P01.37, P02.39

Han, Mei ....................................... P03.10

Han, Yuanyuan ........................... P01.25

Hanes, Douglas...............P03.03, P09.13, P09.15

Hanser, Suzanne .......................OA07.02

Harris, Richard ........OA02.01, OA05.01

Harrison, James ......................... OA05.04

Hartman, Julie............................... P04.13

Harvey, William F..........P05.36, P05.45

Hasick, Gordon .......................... OA02.02

Hazel, Cynthia ............................... P09.30

He, Liyun .................................... P09.25

$\mathrm{He}$, Xiaotian

P02.05
Head, Kathleen ............................ P03.25

Hecht, Frederick M ... OA05.04, P02.22, P05.01, P05.63, P09.31

Hedal-Siegel, Jane ........................ P09.26

Helton, Jeffrey ............................ P06.12

Hempel, Susanne ............P05.60, P07.30

Henry, Brook ............................... P05.50

Heo, In ....................................... P02.23

Heo, Kwang-Ho............................ P02.23

Herman, Patricia .....OA03.04, OA06.01, OA10.03

Hernandez, Maria .....................OA07.02

Hernon, Anne Marie... OA02.04, P05.58

Herrington, Craig.......................... P09.18

Hicks, John .................................. P08.09

Hill, Deborah ................................ P06.09

Hinshaw, Daniel .......................... P07.10

Hlavin, Jennifer .........................OA13.04

Ho, Robin ST ... P02.30, P03.17, P08.12

Hodsdon, Wendy ......................... P01.23

Hoffman, Angela ......................... P04.04

Holden, Selma .............................. P05.29

Hollon, Steven ............................ P05.70

Holmberg, Christine .................... P07.38

Holmes, Holly ................................ P07.25

Hondras, Maria ........................... OA03.02

Hong, Sunghyun .......................... P05.66

Hongbo, $\mathrm{Hu}$.................................. P01.32

Horiba, Yuko ...................P01.10, P03.16

Horwitz, Randy ...............P07.07, P07.08

Hosni, Andressa ............................ P01.36

Hou, Yi ........................................ P01.37

Hourston, Sarah ..............P06.04, P08.05, P09.07

Houston, Mark .............................. P03.25

Hovey, Dean ................................ P09.30

Howell, Shakeilla ......................... P07.16

Howick, Jeremy .............................. P09.10

Hoyt, Katie ................................... P06.09

Hu, Hai ......................................... P02.39

Huang, $\mathrm{Na}$..................................... P02.28

Hubbard, Janice ........................... P04.13

Huerta, Timothy ......................... OA01.01

Hui, Edwin P ................. P02.30, P03.15, P03.17

Hui, Ka-Kit .................................. P07.43

Huiyuan, Zhang ........................... P01.32

Hull, Amanda ............................ P05.16

Hunsinger, Matthew .................... P05.14

Hurwitz, Eric ............................... OA06.03

Hurwitz, Jason T ........................P07.08

Hussain-Alkhateeb, Laith ............. P07.12

Huynh, Alexis ............................ OA 10.04

Hwang, Eui-Hyoung .................... P02.23

Hwang, Jiwon ............................. P02.05

Hwang, Man-Suk............................ P02.23

Hyun, MinKyung......................... P07.45

\section{I}

Ichesco, Eric ................................ OA02.01

Icke, Katja.................................. OA05.02

Ihenacho, Ugonna ........................... P07.23

Ijaz, Nadine.................................. P07.17

Ingenito, Carmine ........................ P05.24

Irvine, Katherine ............................. P09.21 
J

Jackson, Brian.................................. P02.02

Jacques, Jacqueline ...................... P03.25

Jain, Shamini …………………..... P05.50

Janik, Hubert.................................. P04.05

Jenkins, Anita ................................ P01.34

Jensen, Carol................................. P06.12

Jensen, J. Eric .............. OA02.04, P05.58

Jeong, Seong Heon ...................... P07.50

Jerger, Kristin .............. OA07.01, P04.06

Jeste, Dilip ...................................A13.03

Jia, Li-Yan .........P02.18, P02.20, P03.10

Jian-Ping, Liu ................................. P06.05

Jiang, Huili ....... P01.09, P01.13, P01.27

Jisheng, Zhang ............................. P01.35

Jo, Junyoung ....................P02.07, P09.08

Johnson, Angela ………................ P02.08

Johnson, Chris ………………......OA15.03

Johnson, Jill ................................. P07.52

Johnson, Pamela Jo ....................... P07.20

Johnston, Bradley ....................... P03.22

Johnston, Jennifer ........ OA02.04, P05.58

Johnston, Kelly .............................. P09.35

Johnstone, Jeanette ....................... P05.31

Jones, David S .............................P09.17

Jones, Kim ………….................... P02.32

Jones, Mark.................................OA03.02

Jong, Mats....................................OA08.04

Jong, Miek ................OA03.01, OA08.04

Joo, Kristina................................. P05.40

Joswiak, Denise .......................... P07.52

Ju, Dahong..................................... P02.39

Jun, Yang .................................... P08.17

Junkes-Cunha, Maíra ..................... P05.48

Jäger, Seabstian ............................ P01.19

K

Kainuma, Mosaburo ....................... P02.35

Kaiser, Nikoletta ............................ P03.12

Kallen, Rachel ............................OA15.04

Kanda, Tatsuya ............................. P09.19

Kang, Myung Ja ............................ P09.08

Kang, Wang .................................... P01.12

Kaplan, George.............................A10.01

Kaptchuk, Ted J ......... .OA15.01, P09.10

Katz, David................................... OA07.01

Kaufman, Adam ........................... P05.20

Kaufman, Bella............................. P02.04

Keever, Teresa ..........................OA07.01

Kelber, Olaf .................................. P03.06

Kerber, Kathryn ............................. P09.16

Kerppers, Ivo ...................P01.36, P07.51

Kessler, Christian ………….......OA09.04

Khalil, Mohamed .......................... P07.48

Khalsa, Sat Bir .......................... P05.32

Khalsa, Sat Bir Singh................... P05.72

Khan, Sumiya ……………….......OA04.04

Kiat, Hosen .................................. P01.34

Killinger, Lisa..............................0403.02

Kim, Byung-Jun ........................... P02.23

Kim, Daehun....................P02.09, P02.10

Kim, Heeyoung.............................. P02.09

Kim, Hyejung ................................ P02.31

Kim, Hyunho ....................P08.07, P09.08

Kim, Ick Tae.

$\mathrm{P} 02.13$
Kim, Jayoung

$\mathrm{P} 02.31$

Kim, Jungkuk

$\mathrm{P} 08.07$

Kim, Kunhyung

$\mathrm{P} 02.09, \mathrm{P} 02.10$

Kim, Woo Sun...

$\mathrm{P} 07.19$

Kim, Young-Eun ............P02.13, P03.19

Kim, Yun Hee

P03.19

King, Hollis .....

P04.07

Kingsley, Rae ................P05.33, P09.32

Kinney, Mary Ellen.

$\mathrm{P} 07.52$

Kinser, Patricia ... $\mathrm{P} 05.09, \mathrm{P} 05.52$

Klatt, Maryanna........... OA01.01, P05.34

Klee, Dan .......OA13.01, P01.11, P09.04

Klein, Sabine

OA02.03

Klem, Mary Lou........................... P09.35

Knee, Christopher........................ P03.01

Ko, Seok-Jae .....................P02.11, P02.12

Kobayashi, Daisuke ..................... P02.35

Koh, Ho Yeon .............................. P07.50

Koithan, Mary ............................... OA08.01

Kolste, Alison ............................ P07.52

Kong, Junhui...................P01.21, P01.22

Koo, Malcolm ............................... P09.23

Kopelman, Jane .......................... P09.26

Kotfila, Joshua ............................. P06.06

Kraemer, Kristen ........................... P09.05

Kraft, Karin......................P03.06, P04.05

Kratz, Susan................................ P04.16

Kreisberg, Joel ............................... P06.07

Kreitzer, Mary Jo ......................... P07.24

Kua, Eh ........................................... P09.34

Kubo, Ai ....................................... P08.06

Kubota, Toshio .............................. P02.35

Kumar, Abhimanyu ....................OA09.04

Kurisu, Michael ........................... P04.07

Kwekkeboom, Kristine .................. P09.27

Kwon, Nancy .............................. P07.25

Köcke, Janina

OA09.01

\section{$\mathbf{L}$}

Lachance, Josée

Laengler, Alfred

Lai, Lily

P06.08

Laird, Kelsey

$\mathrm{P} 07.21$

Laird, Lance D

P07.28

Lam, Chun Nok ..............P07.22, P07.23

Lam, Lo Kuen Cindy ................... P07.39

Lamb, Joseph .............................. P03.25

Lane, Christianne........................ P05.61

Langer, Ellen ............................... P05.75

Langhorst, Jost..........OA09.01, OA09.03

Lao, Lingxing …......................... P02.39

Lao, Lixing ......................P01.26, P02.02

Lasseter, Nancy ............................ P05.23

Lau, Alexander YL..................... P03.15

Lau, Ho Ying............................... P03.15

Lauche, Romy........................... OA09.01,

OA09.03, P04.03, P05.17, P05.35

Lawrence, Dana ..............P06.09, P06.11

Lawrence, Suzanne ........................ P09.35

Lawson, Karen.............................. P07.24

Lay, Cynthia ................................ P05.40

Lazar, Sara W ................................P01.02

Leach, Matthew ............................ P09.09

Lebensohn, Patricia ...................OA08.01

Lee, Augustine

P05.36
Lee, Bennett................................... P06.15

Lee, Haebeom .............................. P08.07

Lee, Hyangsook ............................. P02.07

Lee, Hye Won .............................. P03.19

Lee, Jennifer ................................ P05.23

Lee, Jihyun ................................... P02.34

Lee, Jung Geun.............................. P07.19

Lee, Myeong Soo ........................ P02.37

Lee, Richard ................................. P07.25

Lee, Robert ................................. OA08.02

Lee, Yongho ................................. P02.34

Lee, Yoon Jae.............................. P02.07

Lee, Yvonne .................................. P05.15

LeFebvre, Ron ............................. P06.09

Leiferman, Jennifer ..................... P09.30

Leininger, Brent ............................. P06.02

Leis, Anne................................... P07.14

Lemaster, Chelsey ....................OA03.04, OA07.02, P05.22, P05.37

Lembo, Anthony J ......................AA15.01

Leonard, Anthony C.....................P09.05

Leonel, Larissa L. S ..................... .P09.06

Lestoquoy, Anna Sophia ............ P05.38, P05.39

Leung, Ping-Chung....................... P03.08

Levy, Ilana ..................................... P03.11

Lewith, George .......... OA08.03, P02.14, P07.26, P07.27, P07.28, P07.29,

P08.08, P08.09, P09.10

$\mathrm{Li}$, Hongtao ................................. P01.14

Li, Jing.P01.09, P01.13, P01.24, P01.27

$\mathrm{Li}, \mathrm{Li}$......................................... P01.21

Li, Lingru.................................... P01.25

Li, Patrick .......................P03.08, P05.41

Li, Qing........................................ P02.01

Li, Weihui....................................

Li, Wen-Yuan... P02.19, P02.21, P07.42

Li, Xun...........................P07.42, P08.13

Li, Yujie...................................... P01.31

$\mathrm{Li}$, Zhigang .................................. P01.31

Lian, Fengmei................ P03.07, P03.13, P03.18

Liang, Ning......P02.18, P02.19, P02.29, P07.42, P09.11

Liang, XingchenP01.09, P01.13, P01.24, P01.27

Liao, Yuan

P02.20

Libermann, Towia A ....................P01.02

Lihong, Zhai ................................ P08.17

Lin, Jue ......................................... P05.49

Lin, Shin .......... P03.08, P05.40, P05.41

Linde, Klaus .............................. OA06.02

Lingam, Vimala ........................... P05.67

Liossi, Christina.........................OA08.03

Lisann, Lauren ............................. P05.75

Liu, Baoyan .................................... P09.25

Liu, Dan ...................................... P01.25

Liu, Hui.......................................... P01.29

Liu, Jia .............................P02.15, P02.16

Liu, Jian-Ping .. P02.18, P02.19, P02.20, $\mathrm{P} 02.21, \mathrm{P} 02.28, \mathrm{P} 02.29, \mathrm{P} 03.04$,

P03.09, P03.10, P03.14, P07.42,

P08.09, P08.10, P08.13, P09.11

Liu, Jianping ..... P03.21, P09.12, P09.14

Liu, Jianxun

$\mathrm{P} 01.34$ 
Liu, Ping ..... P02.39

Liu, Rhianon $\mathrm{P} 02.22$

Liu, Shan $\mathrm{P} 02.15, \mathrm{P} 02.16$

Liu, Tian-Kuang .......................... P02.19

Liu, Tiegang ..... P01.15, P01.17, P02.17

Liu, Tonghua ..... $\mathrm{P} 01.37$

Liu, Wei..... $\mathrm{P} 01.08, \mathrm{P} 03.14$

Liu, Zhaolan .... P02.18, P02.20, P03.09, P03.10

Lohr, Kathleen P07.02

Long, Cynthia P06.11

Longhurst, John ..... OA03.02, P06.09

Lopez, Gabriel $\mathrm{P} 01.30$

Lotz, Fabian. P05.77, P07.25

Lown, Mark OA05.02

Lu, Menghan OA04.01

Lu, Ping ... P01.31

Lu, Qingyi

$\mathrm{P} 03.15$

Lu, Yan. $\mathrm{P} 01.33$

Luberto, Christina...... OA15.04, P05.42, P05.43

Luciano, Alison OA07.01

Luo, Guangbin .... P01.37

Lustig, Robert. P09.31

Lux, Linda .... P07.02

Lyons, Felisha.

$\mathrm{P} 05.34$

Lytvyn, Lyubov $\mathrm{P} 03.22$

Längler, Alfred P06.16, P07.38

M

Ma, En-long

P01.05

Ma, Weiguo

P01.29

Ma, Wenhao

$\mathrm{P} 02.05$

Ma, XiaoYun P01.28

Ma, Xuesheng. $\mathrm{P} 01.37$

Magidson, Jessica $\mathrm{P} 05.42$

Maguen, Shira. OA13.04

Mahant, Sanjay

P03.22

Mahoney, Louise $\mathrm{P} 05.07$

Maiya, Meghan. OA13.03

Maizes, Victoria H .........P07.07, P07.08

Malarkey, William.....................OA01.01

Maletich, Carly .......... OA15.03, P05.44, P05.55, P05.59

Mann, John Douglas

$\mathrm{P} 01.14$

Mannheimer, Steven P04.09

Manor, Brad.... P05.29

Mao, Jun $\mathrm{P} 02.01, \mathrm{P} 03.26$

Marques-Vidal, Pedro................... P07.09

Marshall, Nell .............................. P07.30

Marszalek, Jolanta ....................... P05.45

Martins, Daniel .......... OA06.04, P01.16, P05.46, P05.48, P09.06

Martins, Thiago C ...................... P09.06

Mason, Ashley........ P09.31

Massa, Jennifer . OA11.01

Matsabisa, Motlalepula ............... P01.37

Matter, Ibrahim P04.01

Matthews, Benjamin..................OA11.01

Matuszewicz, Emily ..................... P06.12

May Winges, Donna. P01.25

Mazzardo-Martins, Leidiane ...OA06.04, P05.46, P05.48

McArdle, Patrick

OA04.02
McCabe Ruff, Kelley ................ OA01.03 McDermott, Clare............P07.27, P07.29 McFarland, Christine ................... P09.35 McKee, Diane ............................... P07.01 McKee, Douglas ........................OA04.04 McLeish, Alison .......... OA15.04, P05.43 Mebius, Alexander ....................... P09.10 Medina, Zelina............................ P02.27

Mehal, Wajahat Z......OA04.04, P01.01, P09.01, P09.29

Mehling, Wolf ............OA13.04, P05.63, P05.69

Mehta, Darshan H .........P05.15, P05.18, P06.01

Meier, Larissa P03.12

Memmott, Tabatha ....................OA13.01

Meng, Fengxian ..............P01.29, P01.33

Mengsun, $\mathrm{Yu}$............................... P08.17

Menville, Jesse …........................... P05.73

Messlin, Maja ............................. P07.38

Metzler, Thomas J.....................OA13.04

Meyer, Gil.................................... P02.25

Meyer-Mesterharm, Anouk ......... P05.53

Miake-Lye, Isomi ...........P05.60, P07.30

Michalsen, Andreas .... OA09.04, P03.12

Milak, Carl................................OA07.01

Milbury, Kathrin......................... P05.77

Miller, Alan ................................... P03.25

Miller, Kathleen M.......................P05.18

Miller, Meghan ..........................OA13.01

Miller, Sally ................................ P05.47

Mills, Paul J.............. OA01.04, P05.49, P05.50, P05.64

Millstine, Denise.

Mimura, Masaru ........................... P01.10

Minkalis, Amy ................P04.08, P06.11

Mintie, Daniel............................. P05.72

Mior, Silvano .............. OA06.03, P04.12

Miraglia, Mindy .......................... P05.32

Miri, Andressa ............................ P01.36

Mishima, Rei ................................ P01.18

Mist, Scott.................................. P02.32

Mistiaen, Patriek.......................... P09.10

Mitchell, Braxton.......................OA04.02

Mitchell, Suzanne E .......P05.11, P05.38

Mitchinson, Allison ..................... P07.10

Mittwede, Martin ........................OA09.04

Moher, David............................. OA06.02

Montefusco, Mary ....................... P09.26

Moon, Sung-Pyo ............................ P07.19

Moore, Charity ………................. P07.55

Moore, Michael ........................... P07.28

Moore-Downing, Danae ................ P05.07

Moran, Patricia ...............P05.01, P09.31

Morandi, Antonio ......................OA09.04

Morgan, Laura .............................. P07.02

Morgan, Lucas ............................ P05.36

Morgan, Nani................................ P05.36

Mori, DeAnna ................................ P05.51

Morin, Chantal.............................. P07.31

Morone, Natalia ..............P07.55, P09.35

Moré, Ari O O............................. P01.06

Moskowitz, Judith ..........P05.65, P05.66

Mosquera, Matthew .................... P07.32

$\mathrm{Mu}, \mathrm{Lin}$......................................
Mukae, Haru . P02.35

Mullins, Daniel .........................OA06.02

Munk, Niki ....... P04.09, P04.10, P04.15

Murakami, Aya ............................. P02.35

Murnane, Dale ........................... P07.22

Murphy, Susan...........................OA05.01

Myers, Toshia P09.33

\section{$\mathbf{N}$}

Nahar, Vinayak P05.67

Nahin, Richard............................ P05.73

Napoles, Anna M ....................... P02.22

Nate, KC ...................................... P07.37

Nativ, Ofer.................................. P02.25

Nayback-Beebe, Ann.................. P09.02

Negash, Lily ...................P05.38, P05.39

Neradilek, Moni Blazej ............OA07.04

Neylan, Thomas C....................OA13.04

$\mathrm{Ng}$, Bacon FL .................P02.30, P03.17, P08.11

Nguyen, Ai-Thuan ....................... P01.30

Nguyen, Ashley ............................ P05.40

Nguyen, Teresa ............................ P05.41

Nguyen, Unity ............................ P02.22

Nicklas, Jacinda ............................ P09.30

Nicolaidis, Christina......P06.04, P08.05, P09.07

Nigg, Joel. P05.31

Niles, Barbara ............................... P05.51

Njike, Valentine ..........................OA07.01

Nourani, Bobby ........................... P04.11

Nygaard, Carolyn ........................ P09.13

\section{$\mathbf{O}$}

O'Beirne, Maeve .......................OA06.03

O'Byrne, T. Jamie ........................ P07.40

O'Riordan, Tim .......................... OA08.03

Obeirne, Maeve .......................... P04.12

Ogawa, Keiko ….......................... P01.18

Oh, Dal-Seok ............................... P08.03

Ohm, Jeanne ..... P04.14, P04.18, P04.19

Oken, Barry ............... OA13.01, P01.11, P05.11, P05.31, P09.04

Olejownik, Jennifer ...................OA10.02

Olopade, Olufunmilayo I .............P07.25

Orest, Marianne ............................ P05.68

Ostermann, Thomas... OA09.04, P04.03, P05.53

Osypiuk, Kamila......... OA03.03, P07.33 Owen, Elizabeth .......... OA02.04, P05.58

\section{$\mathbf{P}$}

Paat, Charmagne ........................... P07.06 Pach, Daniel..............................OA05.02 Pagnini, Francesco....................... P05.75 Paillé, Pierre ................................. P06.08 Park, Elyse ................................... P05.42 Park, Gunhyuk ............................ P08.03 Park, Ji Eun .................................. P02.13 Park, Minjung ............................... P07.05

Park, Sang-soo ............................. P07.19

Park, Tae-Young............................. P02.31

Park, Taehwan ........................... P05.28

Park, Young-Bae ........................ P08.07

Park, Young-Jae ............................. P08.07 
Parkin, Patricia P03.22

Parreira, Rodolfo P07.51, P09.06

Parrott, Scott P06.06

Pasalis, Edi $\mathrm{P} 05.32$

Passi, Alvaro

$\mathrm{P} 07.03$

Patel, Reema $\mathrm{P} 05.61$

Patel, Sheila P05.49, P05.64

Patron, Roberto. P07.18

Pearsall, Melanie $\mathrm{P} 05.18$

Percival, Kara P09.22

Perlman, Adam. P06.06

Peterson, Christine Tara ............. P05.49, P05.64

Peterson, Scott $\mathrm{P} 05.49, \mathrm{P} 05.64$

Petkewicz, Jacqueline.................. P05.59

Petronilho, Fabrícia ...................OA06.04

Pfeffer, Raphael ............................ P02.04

Pham, Richard ............................. P05.41

Pham, Son................................... P05.41

Philips, Kamaira ........................... P01.14

Phipps, Stephen ........................... P03.25

Pieper, Carl ................................OA07.01

Pierl, Christiane ........................... P07.02

Pilkonis, Paul ................................. P09.35

Pless Kaiser, Anica...................... P05.51

Plumb, Dorothy ........................... P05.37

Pohl, Elisabeth............................ P07.38

Pohlman, Katherine .... OA06.03, P04.12

Polireddy, Kishore.........P01.03, P01.04, P01.05

Polizzi, Craig.

P05.51

Poon, Simon K

P03.15

Porter, Kristen. OA05.03

Porter, Valencia

P05.49, P05.64

Pressman, Alice

P05.69

Price, Ashley.. P07.06

Price, Cynthia

Price, Lori Lyn

OA13.02

Proulx, Jeffrey

P05.36, P05.45

Pung, Meredith $\mathrm{P} 01.11$ P05.50, P05.64

Purohit, Maulik.

P05.73

Q

Qian, Shen ..

P01.12

Qin, Lingling

P01.37

$\mathbf{R}$

Rabago, David

P04.11

Rahtz, Emmylou .............P07.47, P08.14

Ramelet, Anne-Sylvie ................. P07.54

Ramos, Miriam ............................. P09.22

Rampp, Thomas........................OA09.03

Rangsong, Hui ............................. P08.17

Ray, Mandira ................................ P07.25

Reddy, Deepti ............................... P09.21

Reddy, Sanjay

OA05.04

Redwine, Laura........... OA01.04, P05.50

Reed, Gregory ……....................... P03.20

Reeves, Alexis .............................. P05.69

Rehfeld, Rick A..............P07.07, P07.08

Reichling, Jürgen .......................... P03.06

Reid, Sheila.

P05.68
Reilly-Spong, Maryanne.

P05.28

Reiss, Helene ................................ P07.20

Ren, Jun

$\mathrm{P} 07.42$

Resnick, Kirsten

OA07.02

Reynolds, Jesse

P09.03

Ridge, Damien

P08.04

Rieber, Alyssa.

$\mathrm{P} 07.25$

Righter, Allison

OA11.01

Riley, David.

P08.01

Ring, Melinda .........OA10.02, OA15.03, P07.32

Rivard, Rachael ......OA11.03, OA11.04, P07.10, P07.11, P07.37, P07.46, P07.49, P07.52, P07.53, P08.15, P09.16

Roberts, Rhonda ......... OA11.02, P05.20

Robertsdottir, Gudrun................. P07.15

Robinson, Nicola .......................... P09.11

Rodondi, Pierre-Yves ....P07.09, P07.35, P07.54

Rodriguez, Alejandra

P09.22

Rodriguez, Edgar...

$\mathrm{P} 04.02$

Rolff, Jana.

$\mathrm{P} 01.19$

Roll, Stephanie

OA05.02

Rosas, Ralph F

$\mathrm{P} 01.16$

Rose, Janet $\mathrm{P} 05.33, \mathrm{P} 09.32$

Roseen, Eric.............OA07.02, OA11.02

Rosenberger, Lisa ....... OA07.01, P09.03

Rosenthal, Blake.......................... P08.06

Roth, Anna.................................... P05.54

Rountree, Robert.......................... P03.25

Rubin, Dan.................................... P05.14

Russell, Alexandra....................... P05.70

Ruth, Chris................................... P07.22

Ryan, Kathy ...............................OA04.02

Ryan, Neal .................................. P09.35

Rybarczyk, Bruce ........................ P06.15

\section{S}

Sadeghi, Mohammad Jafar.......... P07.44

Sagee, Avigail................................ P04.01

Saito, Yuri................................... P05.79

Salgado, Afonso .............P01.36, P04.17, P07.51, P09.06

Salm, Daiana................................ P09.06

Salsbury, Stacie ......... OA03.02, P04.13, P07.36

Sanford, Stacy............................. P05.44

Santana, Trilce............ OA05.04, P02.22

Santos, Adair R S ......................OA06.04

Santos, Israel................................ P04.17

Saper, Robert ...........OA03.04, OA07.02, OA11.02, P05.22, P05.37

Sarmento, Fernando..

P09.19

Saslow, Laura .............................. P09.31

Satyshur, Maureen ........................ P05.66

Saul, Bradley ............................... P09.33

Sauve, Anthony A ........................P01.02

Sayre, Nancy ................................. P06.12

Scala, Emmanuelle ...................... P07.35

Schadt, Eric

P05.49, P05.64

Scheffer, Christian ...........P06.16, P06.18

Scheid, Volker .............................. P08.04

Scherban, Alisa..

OA04.04

Schienle, Jessica

P05.07
Schiff, Elad ....... P02.25, P03.11, P04.01

Schillinger, Dean

$\mathrm{P} 02.22$

Schmalzl, Laura.

OA13.03

Schneider, Michael ........P06.09, P06.10, P09.35

Scholkmann, Felix .....................OA02.03

Schroeder, David .......................... P05.14

Schuette, Stephanie ... OA15.03, P05.44, P05.55, P05.59

Schuler, Caitlin ...

P07.49

Schäfer, Miriam ..........................OA09.01

Schöls, Margarita.........................OA09.01

Scott, John G ............................... .P09.17

Secor, Eric ......................P03.24, P09.29

Seely, Andrew ............................. P09.18

Seely, Dugald ..................P03.01, P09.18

Seifert, Georg .................P01.19, P07.38

Selby, Michelle............................. P07.29

Seldenrijk, Ruth..........................OA03.01

Semba, Richard.......................... OA04.02

Sen, Ananda................................OA05.01

Seo, Bok Nam .............................. P02.13

Seto, Sai Wang ............................. P01.34

Shao, Qianfeng ............................ P01.31

Shapiro, Eugene D .......................P05.06

Sharma, Manoj ............................ P05.67

Sharma, Varun ............................. P05.79

Sharpe, Michael ........................... P07.27

Shekelle, Paul .................P05.60, P07.30

Shen, Xueyong ............................ P01.26

Shen, Zhiming .............................. P01.33

Shen, Zhong-Yuan....................... P05.41

Sheng, Guo ................................ P01.12

Sherman, Karen ........................... OA03.04

Shi, Mai ........................................ P03.10

Shi, Peng ........................P01.09, P01.20

Shimazoe, Takao ......................... P02.35

Shin, Byung-Cheul ..................... P02.23

Shinday, Nina ............................ P05.56

Shirai, Tamami ...............P05.57, P07.34

Shoemaker, Richard ................... P05.73

Short, Amy ................................. P09.05

Short, Jack ................................... P07.18

Shue, Sarah ....................P04.09, P04.10

Shuldiner, Alan ..........................OA04.02

Shuldiner, Scott R ........................P08.12

Shuster, Jonathan .......................... P05.23

Sieck, Cynthia............................ OA01.01

Sievers, Patricia ........................... P02.24

Silveri, Marisa ............ OA02.04, P05.58

Silverman, Wendy K....................P05.06

Simmons, Angela ........................ P09.02

Siu, Wing-Sum ............................. P03.08

Sivan, Keren ................................ P04.01

Skidmore, Becky .......................... P03.01

Slattengren, Andrew ....................... P04.11

Slutsky, Jerry ............................... P09.35

Smith, Gina..................................OA07.01

Smith, Kristin .............................. P05.44

Smith, Patricia .............................. P02.33

Sohaibani, Imen ........................... P07.48

Solloway, Michele ........................ P05.60

Sommers, Elizabeth....................OA05.03

Son, Gyungmo ............................. P02.09

Son, Mi Ju ………........................ P03.19 
Sonack, Julie

Sood, Amit.... P05.68

Sossin, Kayla

P05.79

Spelman, Amy

P05.16

Spoo, Michaela $\mathrm{P} 05.77$

Spooner, Chris

OA09.04

Spruijt-Metz, Donna .......P05.61, P05.62

Sroka, Gideon

P04.01

Stadler, Diane ... P06.04, P08.05, P09.07

Stadler, Walter. $\mathrm{P} 07.25$

Stange, Kurt C .............OA10.01, P09.17

Stange, Rainer. P03.12

Stanton, Michael........................... P05.07

Stapelfeldt, Elmar .....................OA09.04

Staples, Julie $\mathrm{P} 05.72$

Steinhubl, Steven R........P05.49, P05.64

Steinle, Nanette ..OA04.02

Stencel, Dianna.

OA15.03

Stephens, Elizabeth $\mathrm{P} 05.14$

Steurich, Justin P03.22

Stewart, Terry P07.18

Stillman, Sarah P05.39

Stites, John P06.09, P06.11

Stoppelman, Nadav..................... P02.25

Streeter, Chris ............. OA02.04, P05.58

Stritter, Wiebke ............................ P07.38

Stuart, Beth ................................. P07.27

Stumpe, Christoph ...................... OA09.03

Su, Chun-Xiang ..............P02.19, P02.29, P08.10

Su, Jing .....

P09.20

$\mathrm{Su}$, Lishan .................................... P01.14

Sufrin, Nathaniel........ OA15.03, P05.44, P05.55, P05.59

Sullivan, Kelli. P05.73

Sun, Jin $\mathrm{P} 02.19, \mathrm{P} 02.21, \mathrm{P} 08.13$

Sun, Lan $\mathrm{P} 01.13, \mathrm{P} 01.27$

Sun, Wen P01.37, P02.39

Sun, Ya'nan ................................ P09.25

Sundberg, Tobias........ OA07.03, P07.12

Sunega, Jean ................................. P03.20

Sung, Hyun Kyung..........P02.06, P07.50

Sunwoo, Yun-Young ................... P02.31

Suter, Marc .................................. P07.35

Suzuki, Kiyoshi .......................... P09.19

Szmulewitz, Russell

P07.25

T

Tam, Jonathan..................P03.08, P05.41

Tan, Chay Hoon ............................ P09.34

Tan, Kay See .............................. P09.26

Tan, Xi ...............P01.21, P01.22, P01.28

Tanaka, Kenji F............................P01.10

Tang, Yinshan............................... P01.31

Tanner-Smith, Emily ................... P05.70

Tanzi, Rudolph E ............P05.49, P05.64

Tarash, Ricardo........................... P02.04

Tardik, George ............................. P03.01

Tauschel, Diethard...........P06.16, P06.18

Taylor, Stephanie....... OA10.04, P05.60, P07.30

Taylor-Piliae, Ruth ...................... P05.47

Thiel, Haymo ............... OA06.03, P04.12

Thoms, Meral .............................. P05.17

Threader, Jennifer

P09.18
Tian, Jiaxing ...................P03.07, P03.13 Tian, Xia ........... P02.03, P02.21, P02.28 Tibbles, Anthony $\mathrm{P} 04.12$

Tilburt, Jon ................................... P07.40

Tippens, Kimberly ....................... P08.01

Tippens, Kimberly M.................. P09.15

Tjen-A-Looi, Stephanie ................... P01.30

Tochterman, Alyssa ..................... P07.41

Tollefson, Michelle ..................... P06.12

Tong, Xiaolin.... P03.07, P03.13, P03.18

Topol, Eric J ...................P05.49, P05.64

Toveg, Miria .............................OA05.04

Treadway, Brittany ....................... P04.02

Treweek, Shaun .......................OA06.02

Trifunovic, Nikola ........................ P01.07

Trocchia, Nicole ............................ P09.26

Trumble, Ericka ........................... P07.10

Tsoi, Kelvin KF..............P02.30, P03.17

Tsukamoto, Marina....................... P01.10

Tsutsui-Kimura, Iku ................... P01.10

Tu, Dahong ................................... P03.10

Tu, Ya ......................................... P01.09

Tucker, Basil............................... P09.09

Twardziok, Monika ..................... P01.19

Twist, Elissa

P04.13

$\mathbf{U}$

Upchurch, Dawn M......................P07.20

Urbano, Jéssica ............................ P04.17

Urrutia, Susana ............................. P02.26

V

Vadodaria, Amisha ....................... P07.56

van de Vijver, Lucy ..................OA03.01

van der Windt, Danielle.............OA06.02

van Dulmen, Sandra .................... P09.10

Van Leuven, Laura........................ P04.11

van Osch, Mara ............................ P09.10

Van Vliet, Marja.........................OA08.04

Victorson, David......... OA15.03, P05.44, P05.55, P05.59, P07.32

Vihstadt, Corrie ......................... P06.02

Vining, Robert ........... OA03.02, P04.13, P07.36

Vinjamury, Manjusha .................. P02.27

Vinjamury, Sivarama Prasad...... P02.27, P04.02

Violet Larson, Jessie ................... P02.33

Vissman, Shannon ....................... P05.46

Vohra, Sunita .............. OA06.03, P04.12

Von Roenn, Jamie ....................... P07.25

W

Waeber, Gérard.............................. P07.09

Wahbeh, Helané ........................OA13.01

Walker, Lynn ................................ P05.70

Wallace, Robert .........................OA03.02

Wallerius, Stephanie.................... P07.49

Wan, Yuxiang ……......... P01.15, P01.17, P02.17

Wang, Chenchen........ OA09.02, P05.36, P05.45, P05.51

Wang, Cong-Cong

P02.28

Wang, Congcong ......

$\mathrm{P} 02.03$

Wang, Ji

$\mathrm{P} 01.25$
Wang, Jie . P01.33

Wang, Juan P01.28

Wang, Li-Qiong P03.14, P07.42

Wang, Lizhen ............................... P01.26

Wang, Mei ..................................... P09.11

Wang, Qi..................................... P01.25

Wang, Qian-fei ............................. P01.25

Wang, Shihwe.............................. P05.37

Wang, Sihan ............................... P01.24

Wang, Ssu-Min ............................... P09.23

Wang, Wenjian ............................ P07.43

Wang, Xuan ................................... P01.17

Wang, Yana ................................ P01.29

Wang, Yanan .............................. P01.33

Wang, Yu......... P01.09, P01.13, P01.24, P01.27

Wang, Yu-Yi .............................. P02.29

Wang, Yuh-Hai............................ P09.23

Wang, Yunhui.............................. P02.17

Wang, Zhijie ................................. P09.20

Warber, Sara .... P07.47, P08.14, P09.17, P09.21

Wardle, Jon.

OA07.02

Wasson, Rachel ........................... P09.05

Watanabe, Kenji .............P01.10, P03.16

Wayne, Peter...........OA03.03, OA09.03, P05.35, P05.56, P07.33

Weeks, John............................... OA10.02

Wegener, Tankred ........................ P03.06

Wei, Lai ......................................... P05.34

Wei, Qi ......................................... P05.77

Weidlich, Christopher ................. P09.02

Weigensberg, Marc.........P05.61, P05.62

Weiguo, Ma ................................. P01.35

Weinberg, Janice ....................... OA03.04

Weiss, Lizabeth ...............P05.49, P05.64

Weiss, Theresa R.......OA04.04, P01.01, P05.06, P09.01, P09.29

Wen, Cheng …….......................... P05.61

Weng, Helen .................................. P05.63

Werner, Maria F............................ P01.06

Wheeler, Amy E...........................P05.18

White, Chris................................... P09.05

White, Laura F ...........................P05.39

White, Peter ................ OA08.03, P07.27

Whitfield, Theodore ................ OA02.04, P05.58

Wieland, L. Susan .....................OA06.02

Willett, Walter ............................OA11.01

Willnauer, Charles ........................ P07.41

Wilson, Kathleen ....... OA01.04, P05.49, P05.50, P05.64

Wilson, Mark.

P06.09

Wilson, Vanessa ........................... P05.11

Wing, David ............................. OA13.03

Winters, David............................... P07.26

Wise, Emaline ............................. P05.34

Wisner, Katherine......................... P05.66

Wisneski, Leonard ....................... P06.12

Witt, Claudia M......OA05.02, OA06.02, OA09.04, OA10.01

Wolever, Ruth............ OA01.03, P04.06, P05.19, P05.20

Wolf, Martin OA02.03

Wolf, Ursula

OA02.03 
Wolfe, Stacie P01.23

Wolinsky, Fredric OA03.02

Wong, Mei Fung Maggle.

P01.09

Wong, Meifeng

Wong, Raimond.

Wong, Samuel YS P03.17, P08.11

Wong, Wendy P02.30, P03.15, P07.39, P08.12

Woo, Jong-Min P06.13

Woodfield, Charles OA02.02

Woods, Gerald P04.04

Woolf, Steven OA10.01

Wruck, Katja OA09.04

$\mathrm{Wu}$, Darong P09.28

Wu, Fan

$\mathrm{P} 01.26$

Wu, Helen P06.17

$\mathrm{Wu}$, Jihong P01.09

$\mathrm{Wu}$, Jimin $\mathrm{P} 07.25$

Wu, Justin CY P03.17, P08.11, P08.12

$\mathrm{Wu}$, Lili...................................... P01.37

Wu, Xin Yin.... P02.30, P03.15, P03.17, P08.11, P08.12

Wyatt, Gwen

OA05.01

$\mathbf{X}$

Xenakis, Lea ................................ P07.06

Xhignesse, Marianne .................... P06.08

$\mathrm{Xia}, \mathrm{Ru}-\mathrm{Yu}$................................ P02.28

Xing, Yanli ................................... P09.20

Xinjing, Yang ............................... P08.17

Xiong, Wen-Jing .......................... P08.02

Xiuyan, Yang ................................ P08.17

$\mathrm{Xu}$, Guangyuan ............................. P01.37

Xu, Mingmin .... P01.09, P01.13, P01.27

$\mathrm{Xu}$, Tunhai ..................................... P01.37

Xue, Pei ....................................... P06.05

Xueqin, Yang.............................. P08.17

Xuezheng, Shang .......................... P01.32

Y

Ya, Tu.

P02.05, P08.17

Yan, Liyi

$\mathrm{P} 02.17$
Yan, Xiaoyi...... P02.18, P02.20, P03.09, P03.10

Yanan, Wang

$\mathrm{P} 01.12$

Yang, Chang Sop ........................ P02.13

Yang, EunMee ............................ P07.06

Yang, Guo-Yan............................ P02.29

Yang, Jia ...................................... P02.39

Yang, Kiyoung ........................... P02.10

Yang, Narae ....................P02.06, P02.31

Yang, Shih Wei ............................ P09.23

Yang, Xinjing ................P01.09, P01.13, P01.24, P01.27, P02.05

Yang, Xueqin. $\mathrm{P} 02.05$

Yang, Zhi Min

P07.39

Yarandi, Hossein

P05.25

Yardley, Lucy

OA08.03

Yeh, Gloria ....OA15.01, P05.29, P05.56

Yerges-Armstrong, Laura...

OA04.02

Yetz, Neil

OA13.03

Yin, Yang

$\mathrm{P} 01.25$

Yingge, A $\mathrm{P} 02.05$

Yoo, Ho-Ryong ...............P07.19, P09.36

Yoo, Jeong-Eun ..............P07.19, P09.36

Yoshino, Tetsuhiro ....................... P03.16

Yost, Kathleen ……...................... P07.40

Yu, Bai........................................... P01.32

Yu, Changhe .............................. P09.25

Yu, Elaine ...................................... P09.24

$\mathrm{Yu}, \mathrm{He} . . . \ldots \ldots . . . . . . . \mathrm{P} 01.15, \mathrm{P} 01.17, \mathrm{P} 02.17$

Yu, Lan ....................................... P09.35

Yu, Miao........... P01.09, P01.13, P01.27

$\mathrm{Yu}$, Qiuyun ...... P01.09, P01.13, P01.24, P01.27

$\mathrm{Yu}$, Ruoxi

$\mathrm{P} 01.25$

Yu, Xiaotong $\mathrm{P} 03.18$

Yue, Shu-Jin P02.19, P08.10

$\mathbf{Z}$

Zafonte, Ross

P05.73

Zângaro, Renato

$\mathrm{P} 07.51$

Zecco, Glenn

P09.26

Zera, Chloe

P05.29

Zhang, Bodi
Zhang, Chuntao P01.09, P01.13, P01.27

Zhang, Honghong .........................P01.29, P01.33

Zhang, Huimin $\mathrm{P} 03.04$

Zhang, Jianping ......................... P01.33

Zhang, Kang ..... P02.19, P03.04, P09.11

Zhang, Li-Juan............................. P02.28

Zhang, Ruixin ............................. P01.26

Zhang, Weijun ............................. P07.43

Zhang, Weimin ............ OA11.01, P07.06

Zhang, Wenyue............................ P02.05

Zhang, Xuhui ..................P01.13, P01.27

Zhang, Yan .....................P01.25, P07.41

Zhang, Ying .................. P01.05, P07.42, P08.13

Zhang, Yunjiao ..............P02.18, P02.20, P03.09, P03.10, P08.13

Zhao, Baixiao P02.39

Zhao, Bingcong .............P01.13, P01.24, P01.27

Zhao, Huihui $\mathrm{P} 01.28$

Zhao, Linlu P09.18

Zhao, Ruizhi

P05.28

Zheng, Luyu $\mathrm{P} 01.25$

Zheng, Zian....... P01.15, P01.17, P02.17

Zhong, Sen. $\mathrm{P} 03.05$

Zhou, Fen.......... P03.21, P09.12, P09.14

Zhou, Jingxin.............................. P01.37

Zhou, Jun ...................................... P08.10

Zhou, Li ......................................... P02.18

Zhou, Xiaojing............................ P03.23

Zhu, Maoxiang …......................... P02.39

Zhu, Xuewei ................................ P01.14

Ziadi Lotfabadi, Maryam ............. P07.44

Zick, Suzanna ...........OA02.01, OA05.01

Ziea, Eric TC ................. P02.30, P03.17, P08.11

Ziea, Tat Zhi Eric....................... P07.39

Zukeyama, Niina ......................... P02.35

Zwarenstein, Merrick ................OA06.02

Zwickey, Heather .......OA08.02, P06.04, P08.05, P09.07, P09.13, P09.15 